\title{
Spectral mismatch and its effect on artificial solar light source under standard test conditions
}

\author{
Gao Sheng ${ }^{1}$, Lu Xiaodong ${ }^{1}$, and Lun Shuxian ${ }^{2, *}$ \\ ${ }^{1}$ Key Laboratory of Microelectronics Process Control of Liaoning Province, Liaoning, 121000, China; \\ ${ }^{2}$ Liaoning Province Photovoltaic Power Control and Integration Engineering Technology Research Center, Liaoning, 121000, China
}

\begin{abstract}
Under standard test conditions, the spectral irradiance of artificial solar light sources and the spectral response of photovoltaic devices are important factors that affect the accuracy of device test results. This paper takes the standard solar spectrum AM1.5 as a reference, and calculates the difference between the four commonly used artificial solar light sources (Arc Lamp, Q-Flash, Q-Flash w and ELH) and the standard solar spectrum AM1.5 from the perspective of spectral mismatch. The spectral mismatch factor and the output parameters of the amorphous silicon cell under the irradiation of these light sources. Calculations show that the spectral mismatch factor of Arc Lamp is only 1.005, which matches the standard solar spectrum AM1.5 best. Due to the effect of spectral mismatch, the output parameters of amorphous silicon cells will change significantly after different artificial light sources irradiate them.
\end{abstract}

\section{Introduction}

With the continuous expansion of the application field of photovoltaic power generation technology and the increasing complexity of applications, the stability and reliability of photovoltaic power generation systems have become one of the main obstacles hindering the further development of this technology [1]. Solar cells are the core components of photovoltaic power generation systems. They mainly work in series and parallel components in photovoltaic power generation systems. Therefore, whether the parameters of the solar cells in the components match or not will have a direct impact on the stability and reliability of the components. And ultimately affect the performance of the entire photovoltaic power generation system. In the production process of photovoltaic modules, the main method to ensure the matching of battery parameters in the module is to classify the performance of solar cells based on the output parameters of the cells under standard test conditions (STC), so how to accurately obtain the solar cell output under STC Parameters have always been a hot issue in the field of photovoltaic power generation $[2,3]$.

At present, the widely accepted and adopted battery cell test conditions are the standard test conditions (STC) formulated by the IEC (International Electrotechnical Society), which stipulates [4]: The spectral distribution of the light source is AM1.5, and the radiation intensity is $1000 \mathrm{~W} / \mathrm{m}^{2}$; the test cell has a uniform temperature distribution, and the temperature should be $25^{\circ} \mathrm{C}$. Research shows that [5]: If the test environment temperature and the temperature of the temperature control system are strictly controlled at $25^{\circ} \mathrm{C}$, the battery temperature and its distribution during the test basically meet the STC requirements of the IEC. Because the light source used in the battery test process is a variety of artificial solar light sources, its spectral properties are not consistent with AM1.5, so under the condition that the temperature is accurately controlled, the test error of the battery output parameters is mainly caused by the difference between the test light source and AM1.5 The mismatch between the spectra leads to [6].

In the study of the spectral mismatch between the test light source and AM1.5, the mismatch factor is mainly used to describe the degree of mismatch between the two spectra [6] and to correct the error caused by the output parameters of the battery or component due to the spectral mismatch [7]. For example: Field et al. studied the relationship between the spectral mismatch factor and the radiation wavelength of the test light source [8]; J. Hohl-Ebinger et al. studied how to use the spectral mismatch factor to correct the output parameters of the battery under standard test conditions [9]; Y. Imai et al. studied the relationship between the performance degradation of photovoltaic modules and the spectral mismatch factor under different radiation levels [10]; M.Tsuji et al. used the spectral mismatch factor to predict the output parameters of photovoltaic modules under practical conditions [11]. At present, most studies on the problem of spectral mismatch use the AM1.5 spectrum as a reference to discuss how to improve the test accuracy of a single artificial light source under standard test conditions and predict the degradation of battery or component output parameters under actual use conditions.

This article uses the standard AM1.5 solar spectrum as a reference, uses the spectral response curve of a

\footnotetext{
*Corresponding author: jzlunzi@163.com
} 
crystalline silicon cell, discusses the spectral mismatch of four common artificial light sources, and combines the battery output parameter analysis to select artificial light sources and improve artificial light sources under standard test conditions. The test accuracy of the light source provides a certain reference.

\section{Battery models and spectral mismatch assessment methods}

The numerical calculation method, calculation process method, grid division scheme and data processing process used in this paper are all consistent with the literature $[5,12,13]$.

\subsection{The battery model}

The structural model of the amorphous silicon cell is shown in Figure 1. Where h1 represents the thickness of antireflection film (ARC, select $\mathrm{SiN}_{\mathrm{x}}$ film), h2 represents the active layer thickness, $\mathrm{h} 3$ represents the buffer layer (BL, select $\mathrm{ZnO}$ thickness) and h4 represents the back mirror thickness of $\mathrm{Ag}(\mathrm{Ag} \mathrm{BR})$ respectively. $\mathrm{L}$ represents the period of texture structure of the woven amorphous silicon cell, and $\theta$ is the texture angle. During the calculation $h 1=75 \mathrm{~nm}, h 2=500 \mathrm{~nm}$

$$
\begin{aligned}
& h 3=100 \mathrm{~nm} \quad, \quad h 4=200 \mathrm{~nm}, \quad \theta=54.7^{\circ}, \\
& L=450 \mathrm{~nm} .
\end{aligned}
$$

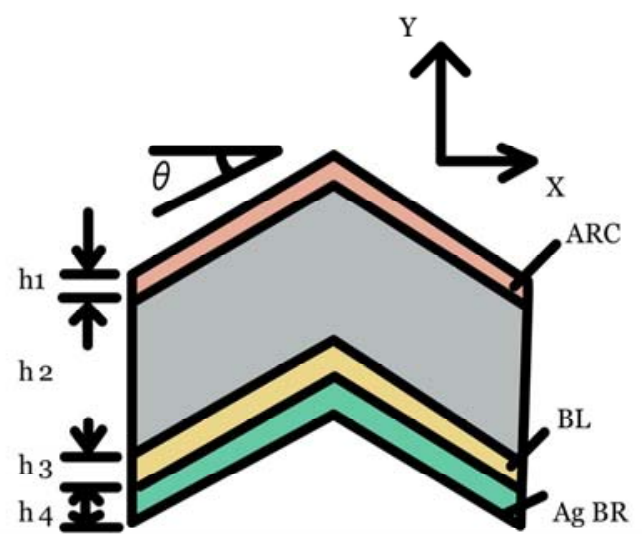

Fig. 1. Amorphous silicon cell structure model.

\subsection{Methods for evaluating spectral mismatches}

The four artificial light sources selected includeXenon arc lamp (Arc Lamp), Q-Flash lamp, Q-Flash w lamp and ELH lamp, of which the xenon arc lamp (Arc Lamp) is a gas discharge lamp with high brightness and a spectrum close to AM1.5 spectrum is the most commonly used light source in solar battery testing; QFlash lamp and Q-Flash w lamp are two kinds of flashes, which can output high-energy light instantly, and are mostly used in the test process of battery or component transient characteristics; ELH lamp is a kind of projection lamp widely used in simple solar battery test system. In the numerical calculation, the spectral data of various artificial light sources and the optical parameters of materials come from professional data websites [14]. Figure 2 shows the comparison between the standard AM1.5 spectrum and the spectrum of four artificial light sources. Obviously, from the perspective of the energy density envelope curve according to the wavelength distribution and the characteristic spectral peak, the spectrum of these artificial light sources is significantly different from the AM1.5 spectrum. From the perspective of the solar cell photoelectric conversion process, the deviation of the spectrum of the artificial light source from the AM1.5 spectrum will inevitably cause the output parameters of the battery to drift, which in turn affects the test accuracy of the output parameters.

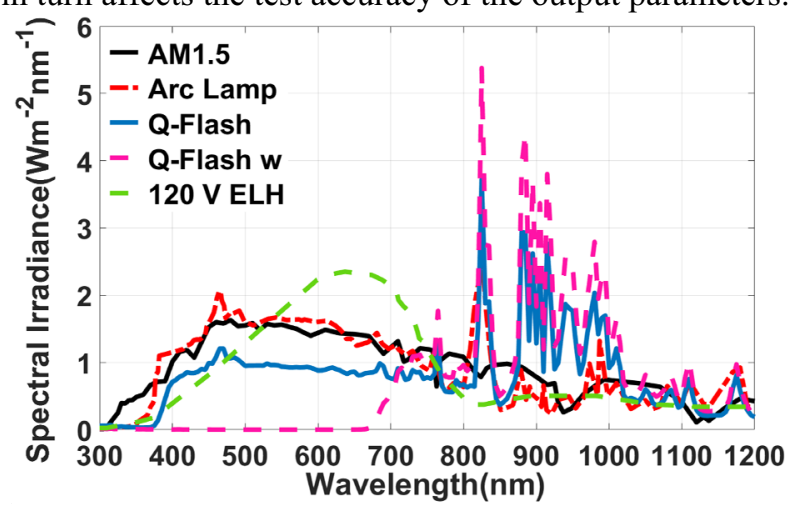

Fig. 2. Comparison result of spectral irradiance.

The spectral mismatch factor (MM) is usually used to describe the degree of spectral mismatch between artificial light sources and standard sunlight. Definition of MM [15]:

$$
M M=\frac{\int_{a}^{b} E_{s}(\lambda) S_{s}(\lambda) d \lambda}{\int_{a}^{b} E_{t}(\lambda) S_{s}(\lambda) d \lambda} \frac{\int_{a}^{b} E_{t}(\lambda) S_{t}(\lambda) d \lambda}{\int_{c}^{d} E_{s}(\lambda) S_{t}(\lambda) d \lambda}
$$

In formula (1), $E_{s}$ and $E_{t}$ are the standard solar spectrum (AM1.5) and the artificial light source spectrum, respectively; $S_{s}$ and $S_{t}$ are the spectral response of the reference battery and the test battery respectively; $a$ and $b$ are the spectral absorption limits of the reference battery, respectively; $c$ and $d$ are the spectral absorption limits of the test battery, respectively. Considering that the output parameters of the reference battery are accurate under standard sunlight irradiation, this article discusses the spectral mismatch problem of artificial light sources based on this situation as the standard, so in the simulation process, a reasonable assumption is often used it is assumed that the spectral response of the reference cell under standard solar radiation is 1 , that is $S_{s}=1$, [15]. Considering $J=\int E(\lambda) S(\lambda) d \lambda(E$ is the irradiation spectrum, $S$ is the spectral response). Then Equation (1) will be transformed into:

$$
M M=\frac{E_{s s}}{E_{t t}} \frac{J_{t t}}{J_{s t}}
$$


In formula (2), $E_{s s}$ and $E_{t t}$ are the total radiant energy of standard solar radiation and artificial solar radiation respectively; $J_{s t}$ is the photocurrent density of the cell under standard solar irradiation; $J_{t t}$ is the photocurrent density of the battery was measured under the irradiation of artificial light source. In the actual cell test process, if $M M>1$, it means that the output optical power of the artificial light source is too large; if $\mathrm{MM}<1$, it means that the output optical power of the artificial light source is too small; if $\mathrm{MM}=1$, it means that the artificial light source matches well with AM1.5.

\section{Results and discussion}

\subsection{Methods of evaluating spectral mismatchs}

When light is incident on the amorphous silicon solar cell, the metal mirror on the back surface can effectively prevent the light from leaking from the bottom surface of the amorphous silicon cell. Therefore, from the perspective of energy conservation, the incident light will finally be decomposed into two parts: absorbed light and reflected light. The absorbed incident light will generate photo-generated carriers inside the amorphous silicon cell and form a photocurrent. Figure 3 shows the distribution of the absorption coefficient and reflection coefficient of the amorphous silicon cell in Figure 1. Note: The spectral response in formula (1) is closely related to the absorption coefficient of the device, but it is not completely consistent. It can be seen from Figure 3 that in the short wavelength range $300 \mathrm{~nm} \sim 400 \mathrm{~nm}$, because the refractive index of the amorphous silicon battery material itself is relatively calibrated, the reflection of incident light is also weak, so the absorption coefficient of the amorphous silicon battery in this wavelength band is relatively large; in the wavelength range $500 \mathrm{~nm} \sim 800 \mathrm{~nm}$, because the refractive index of the amorphous silicon battery material increases with the increase in wavelength, the absorption coefficient of the amorphous silicon battery in this band is relatively small; within the range $800 \mathrm{~nm} \sim 1200 \mathrm{~nm}$, the absorption coefficient of amorphous silicon material is very small.

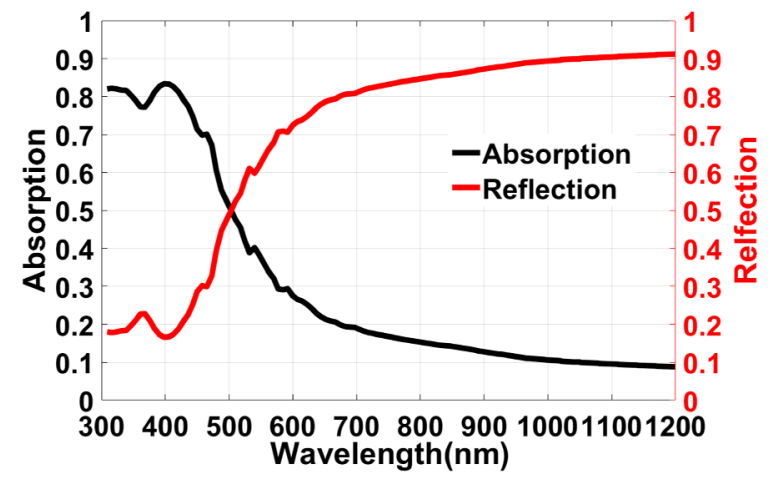

Fig. 3. Light absorption and reflection of amorphous silicon solar cells.

\subsection{Mismatching factors of four kinds of artificial light sources}

Table 2 shows the total photocurrent density after AM1.5 and four kinds of artificial light sources irradiate amorphous silicon cells. It can be seen from Table 2: The total photocurrent density of the amorphous silicon cell irradiated by the Arc Lamp and $120 \mathrm{~V}$ ELH artificial light sources is less different from the total photocurrent density of the AM1.5 light source irradiated by the amorphous silicon cell, while the Q-Flash the total photocurrent density of amorphous silicon cells irradiated by artificial light sources of type and Q-Flash $\mathrm{W}$ is quite different from the total photocurrent density of amorphous silicon cells irradiated by AM1.5 light source.

Table 1. Photocurrent density of amorphous silicon solar cells under various spectra.

\begin{tabular}{|c|c|}
\hline Light source & $\begin{array}{c}\text { Total photocurrent } \\
\text { density } \\
\left(\mathbf{m A} \cdot \mathbf{c m}^{-2} \mathbf{)}\right.\end{array}$ \\
\hline AM1.5 & 12.0420 \\
\hline Arc Lamp & 12.2585 \\
\hline Q-Flash & 9.5220 \\
\hline Q-Flash w & 5.8421 \\
\hline 120 V ELH & 10.6636 \\
\hline
\end{tabular}

Using formula (2), the total mismatch factor MM value of the four artificial light sources can be calculated, as shown in Table 2. It can be seen from Table 2: Because $\mathrm{MM}>1$ and $\mathrm{MM}<1$ have mutual compensation effects, the relationship between the total mismatch factor $\mathrm{MM}$ of the four artificial light sources is: $\mathrm{MM}_{\mathrm{Q}}$ Flash $\mathrm{w}>\mathrm{MM}_{\mathrm{Q}-\text { Flash }}>\mathrm{MM}_{120 \mathrm{~V} \text { ELH }}>\mathrm{MM}_{\text {Arc Lamp, }}$, and the test accuracy is just the opposite.

Table 2. Mismatching factors of four kinds of artificial light sources.

\begin{tabular}{|c|c|}
\hline Light source & Total MM \\
\hline Arc Lamp & 1.010 \\
\hline Q-Flash & 0.877 \\
\hline Q-Flash w & 0.636 \\
\hline 120 V ELH & 0.922 \\
\hline
\end{tabular}

\subsection{Changes in output parameters of crystalline silicon cells due to spectral mismatch}

The output parameters of solar cell mainly include: short-circuit current (Isc), open-circuit voltage (Voc), short-circuit current density $\left(\mathrm{J}_{\mathrm{SC}}\right)$, filling factor $(\mathrm{FF})$, conversion efficiency $(\eta)$, etc. Table 3 shows the output 
parameters of amorphous silicon cells irradiated by AM1.5 and four kinds of artificial light sources.

Table 3. Output parameters of amorphous silicon cells irradiated by four kinds of artificial light sources.

\begin{tabular}{|c|c|c|c|c|}
\hline $\begin{array}{c}\text { Light } \\
\text { source }\end{array}$ & $\begin{array}{c}\text { Jsc } \\
(\mathbf{m A} \cdot \mathbf{c m}-\mathbf{2})\end{array}$ & $\begin{array}{c}\text { Voc } \\
(\mathbf{V})\end{array}$ & $\begin{array}{c}\mathbf{F F} \\
(\mathbf{\%})\end{array}$ & $\begin{array}{c}\eta \\
(\mathbf{\%})\end{array}$ \\
\hline AM1.5 & -7.1788 & 1.0764 & 87.5751 & 8.1788 \\
\hline $\begin{array}{c}\text { Arc } \\
\text { Lamp }\end{array}$ & -7.4038 & 1.0775 & 87.6107 & 8.3917 \\
\hline Q-Flash & -4.5881 & 1.0643 & 87.5135 & 5.7148 \\
\hline $\begin{array}{c}\text { Q-Flash } \\
\text { w }\end{array}$ & -0.7378 & 1.0177 & 87.3626 & 1.0389 \\
\hline $\begin{array}{c}\text { 120 V } \\
\text { ELH }\end{array}$ & -5.9707 & 1.0708 & 87.7204 & 7.0568 \\
\hline
\end{tabular}

\section{4 conclusion}

In this paper, the mismatch factors between the spectra of four different artificial solar light sources and the standard solar spectrum AM1.5 were studied, and the variation of the output parameters of amorphous silicon cells caused by the spectral mismatch was given. The main conclusions are as follows: the four kinds of artificial solar light sources all have different degrees of mismatch with the standard solar spectrum AM1.5: the Arc Lamp type artificial light source has the minimum MM value of 1.01 , which is the best match with the standard solar spectrum AM1.5; the maximum MM value of Q-Flash w artificial light source is 0.636 , and the mismatch between Q-Flash $\mathrm{w}$ and standard solar spectrum AM1.5 is the most serious. Under the irradiation of four kinds of artificial light sources, the four output parameters of crystal silicon cells deviate to different degrees. By integrating the absolute error data, it can be seen that the deviation of the four output parameters of Arc Lamp artificial light source is relatively small. Q-Flash w type artificial light source of four output parameters deviation is relatively large.

\section{References}

1. Michal O. Estimating Effects of Individual PV Panel Failures on PV Array Output[J]. IEEE Transactions on Industry Applications, 2018, PP:1-1.

2. Barduzza G, Pavanello D, Galleano R,et al. Calibration procedure for solar cells exhibiting slow response and application to a dye-sensitized photovoltaic device[J]. Solar Energy Materials \& Solar Cells, 2017, 160(Complete):418-424.

3. Shanshan Chen, Saeid Gholami Farkoush, Sebastian Leto. Photovoltaic cells parameters extraction using variables reduction and improved shark optimization technique[J]. International Journal of Hydrogen Energy, 2020, 45(16): 10059-10069.

4. Woyte A, Nijs J, Belmans R. Partial shadowing of photovoltaic arrays with different system configurations: literature review and field test results[J]. Solar Energy, 2003, 74(3):217-233.

5. Xiaodong Lu, Yukuo Li, Shuxian Lun. Uncertainty of output parameters of crystal silicon cells under standard test conditions[J]. China science, 2019, 049(012): P.1523-1533. (in Chinese)

6. Müllejans H, Ioannides A, Kenny R, et.al. Spectral mismatch in calibration of photovoltaic reference devices by global sunlight method[J], Measurement Science and Technology, 2005, 16: 1250-1254.

7. Ye S, Chen B, Y Cheng, et al. Resolving Spectral Mismatch Errors for Perovskite Solar Cells in Commercial Class AAA Solar Simulators[J]. Journal of Physical Chemistry Letters, 2020, 11(10): 3782-3788.

8. Field H, Emery K. An uncertainty analysis of the spectral correction factor[C]. Proceedings of $23 \mathrm{rd}$ IEEE Photovoltaic Specialists Conference, Louisville, Kentucky, USA, 1993: 1180-1187.

9. Hohl-Ebinger J, Warta W. Uncertainty of the spectral mismatch correction factor in STC measurements on photovoltaic devices[J]. Progress in Photovoltaics: Research and Applications, 2011, 19: 573-579.

10. Imai Y, Chanfana J, Kawano Y, et al. Description of performance degradation of photovoltaic modules using spectral mismatch correction factor under different irradiance levels[J]. Renewable Energy, 2019, 141: 444-450.

11. Tsuji M, Chantana J, Nakayama K, et al. Utilization of spectral mismatch correction factor for estimation of precise outdoor performance under different average photon energies[J]. Renewable Energy, 2020, 157.

12. Xiaodong Lu, Yukuo Li, Shuxian Lun, et al. High efficiency light trapping scheme used for ultrathin cSi solar cells[J], Solar Energy Materials and Solar Cells, 2019, 196: 57-64.

13. Xiaodong Lu, Xinxin Wang, Jie Gao, et al. Twocavity light-trapping scheme used in ultrathin c-Si solar cells[J], Optics Letters, 2018, 43(19): 47314734.

14. (http://www.pvlighthouse.com.au/refractive-indexlibrary).

15. Mambrini T, Dubois A. M., Longeaud C, et.al. Photovoltaic yield: correction method for the mismatch between the solar spectrum and the reference ASTMG AM1.5G spectrum[J], EPJ Photovoltaics, 2015, 6: 60701 . 